\title{
Diversitätssensible Öffentlichkeitsarbeit: Herausforderungen und Möglichkeiten
}

Prasanna Oommen ${ }^{1}$ gilt als Expertin im Bereich diversitätssensible Öffentlichkeitsarbeit und diskutiert im folgenden Beitrag mit Julia Hauck, Agentin für Diversität und interkulturelle Bibliotheksarbeit der Ernst-Abbe-Bücherei Jena, die Herausforderungen und Möglichkeiten für Bibliotheken. Dem nun folgenden Interview wird als Leitsatz der Artikel 27 der Allgemeinen Menschenrechte vorangestellt:

Jeder hat das Recht, am kulturellen Leben der Gemeinschaft frei teilzunehmen, sich an den Künsten zu erfreuen und am wissenschaftlichen Fortschritt und dessen Errungenschaften teilzuhaben. (Artikel 27, Allgemeine Erklärung der Menschenrechte, 1948)

Julia Hauck: Was heißt für Sie diversitätsorientierte Öffentlichkeitsarbeit? Was ist das Ziel?

Prasanna Oommen: Ich verstehe unter diversitätssensibler Öffentlichkeitsarbeit vor allem ein gutes Zusammenspiel zwischen interner und externer Kommunikation. Das heißt, diversitätssensible Öffentlichkeitsarbeit bedeutet eigentlich nicht, dass man singulär die richtige Sprache benutzt, sich mit dem Zielgruppenbegriff auseinandersetzt - all diese Dinge muss man natürlich auch tun -, sondern in erster Linie geht es um eine Haltungsüberprüfung. Und es gilt: Man kann keine gute externe Öffentlichkeitsarbeit machen, wenn die interne Kommunikation nicht funktioniert.

Die Voraussetzung für eine diversitätssensible Öffentlichkeitsarbeit ist, dass man das Wissen der gesamten Institution mit einbezieht. Das ist aber in den Hierarchien bisher nicht mitgedacht. Öffentlichkeitsarbeiter*innen bekommen oft als letzte das Thema, welches herausgegeben werden soll, auf den Tisch. Sie werden nicht oft in die Programmentwicklung oder Personalplanung einbezogen. Sie sollen die Stellenanzeigen schreiben, aber werden nicht inhaltlich einbezogen, ob solch eine Ausschreibung überhaupt Sinn macht für die Stelle. Sie bekommen die Informationen zur Ausstellung, zum Konzept oder zur Veranstaltung und müssen dann in relativ kurzer Zeit einen Flyer entwickeln. Diese operativen Probleme gibt es.

1 Mehr Informationen finden sich auf der Homepage von Prasanna Oommen: www.prasannaoommen.de.

๖ Open Access. (C) 2021 Julia Hauck \& Prasanna Oommen, publiziert von De Gruyter. (๕)BY Dieses Werk ist lizenziert unter der Creative Commons Attribution 4.0 Lizenz. 
Und was dabei unterschätzt wird, ist, dass Diversitätssensibilität in einer Institution heißt, ich muss mein Leitbild, meine Haltung, mein Selbstverständnis innerhalb der Organisation überprüfen. Dies sollte auch ganz klassisch top down verordnet werden. Es müssen natürlich alle bottom up an Bord geholt werden, aber es muss vor allem an der Spitze gewollt sein. Übrigens gilt das auch für die Methode des bereichsübergreifenden Arbeitens.

Ich bin vom Herzen her und von Hause aus Pressesprecherin, das heißt, ich kenne diese Abläufe seit 20 Jahren aus den verschiedensten Bereichen. Nach diesen Erfahrungen kann ich sagen, dass es einen handfesten Grund dafür gibt, warum die Öffentlichkeitsarbeit, gerade in der Privatwirtschaft, immer direkt der Geschäftsführung zugeordnet ist. Diese Personen müssen schließlich die ureigenen Interessen der Institution umsetzen. Wenn die Institution nicht die Haltung hat, diversitätssensibel kommunizieren zu wollen und sich auch nicht darüber verständigt, was damit gemeint ist, dann kann die Öffentlichkeitsarbeit das nicht retten.

Julia Hauck: Die interne Struktur ist auf jeden Fall die Voraussetzung für die ganz praktischen Sachen. Ich möchte gern ein Praxis-Thema aufgreifen: die Zielgruppen. Sie haben schon gerade gesagt, dass man sich mit dem Begriff auseinandersetzen muss. Was für Zielgruppen stehen in der Regel bei einer diversitätssensiblen Öffentlichkeitsarbeit im Fokus?

Prasanna Oommen: Also für mich ist das Wort Zielgruppen total Eighties. Als ich vor zwanzig Jahren beim Fernsehen als Pressesprecherin angefangen habe, sprach man noch von Zielgruppen zwischen 19 und 49 Jahren. Zielgruppen sind aber heute extrem diversifiziert. Der Fokus sollte vielmehr auf Bildungsvoraussetzungen, Milieus, pluralen Identitäten liegen, immer dem Prinzip der sozialen Klasse untergeordnet.

Ich spreche eigentlich auch nicht von Zielgruppen, sondern von Besucher*innen und Nicht-Besucher*innen. Dann reden wir nämlich darüber: Wissen wir genügend über unsere Nicht-Besucher^innen? Über unser Stammpublikum wissen wir viel. Die Kultureinrichtungen, insbesondere auf den Führungsebenen, sind sehr oft mit der Frage beschäftigt: „Wie halten wir unser Stammpublikum?“ Das ist grundsätzlich eine berechtigte Frage. Da stoßen wir aber in bestimmten Diskussionen sehr stark mit den Menschen zusammen, die sich als Aktivistinnen im Bereich Öffnung betätigen - zu Recht.

Das Stammpublikum zu halten, bedeutet, sich anzuschauen: Welche Formate brauchen wir dafür? Welche funktionieren, welche eher nicht? Womit wir uns aber nicht oder nach wie vor viel zu selten in dieser Diskussion beschäftigen, ist die Frage: Warum wollen wir andere Besucher`innen haben, was ist unser Auftrag? Was ist unser Auftrag als eine Institution? Ich meine hier nur Institutio- 
nen, die aus Steuermitteln finanziert werden, die sich um ihre Relevanz sorgen und versuchen, den Anschluss nicht zu verpassen. Und wenn wir da über Zielgruppen reden, dann würde ich sagen, die Institutionen beschäftigen sich zu wenig damit, was die potenziellen Besucher`innen, die im Moment noch NichtBesucher*innen sind, tun, wenn sie nicht bei uns sind. Wenn sie nicht in unserer Stadtbibliothek oder in unserem Museum oder Theater sind, wo sind sie dann? Womit beschäftigen sie sich? Wer weiß, womit sie sich beschäftigen? Und wer ist vielleicht in meiner Institution längst angestellt und könnte das wissen? Das ist manchmal eben nicht die Kuratorin, die Bibliotheksleitung oder die Archivarin, sondern das kann sehr oft auch die Person sein, die in der Hierarchie ganz weit unten ist, nämlich die Schließerin, die Ordnerin, das Sicherheitspersonal, das Putzpersonal.

Julia Hauck: Man muss also analysieren, wer noch nicht kommt. Oftmals wird dann der Aspekt der Teilhabe in den Vordergrund gestellt. Als öffentliche Einrichtung sollte man sich darum bemühen, eben die - und das nennt man im Bibliothekssprech ja manchmal - bibliotheksfernen oder nicht-affinen Menschen zu gewinnen.

Prasanna Oommen: Alles Vorurteile! Den Begriff der bildungsfernen Familien liest man leider immer wieder in Evaluationsberichten oder in Antragspapieren. Das ist aber nur eine Annahme. Worüber wir reden müssen, ist die sozioökonomische Benachteiligung. Aber wir können nicht - und das ist ein ganz großer Irrtum - davon ausgehen, dass Menschen, die nicht in unsere Einrichtungen kommen, nicht kultur-affin sind. Weil wir eben nicht genug über deren kulturelle Praxis wissen. Es gibt im Bereich Kulturelle Bildung - und das betrifft die Stadtbibliotheken ja besonders - ganz tolle Projekte in den letzten Jahren, die zeigen: Wenn wir uns interessieren: für die sogenannten Zielgruppen, für die Nicht-Besucher^innen, dann stellen wir plötzlich fest, dass sie in ihren Communitys sehr viel kulturell praktizieren. Diese Praxis passt nur nicht in unseren Kanon, in unseren Horizont, in das, was wir verstehen. Also ich sage wir und meine damit uns, als Kultur-Bourgeoisie. Ich komme aus einem bildungsbürgerlich geprägten Milieu. Meine Eltern sind zwar in dieser Gesellschaft Bildungsaufsteiger^innen gewesen, weil sie aus einem anderen Land kamen, dann hier ihre Kinder bekommen haben und von vorne beginnen mussten. Aber wir waren bereits so geprägt, dass Kultur relevant war und dass wir uns per musisch-künstlerischer Förderung in mindestens zwei Kulturen beheimaten. In anderen Communitys oder Familien ist das so nicht unbedingt möglich - aus finanziellen, strukturellen oder sprachlichen Gründen. Aber anzunehmen, dass diese Menschen nicht kultur-affin sind, ist eine große Unterstellung. Und hier müsste es mit der Diversitätssensibilität los gehen. 
Julia Hauck: In vielen Bibliotheken ist man aber längst so weit, dass als nicht bibliotheks-affin auch Menschen gelten, die gerade mitten im Leben stehen und die mit Familie und 40-Stunden-Job wenig Zeit haben. In den letzten Jahren ging es oft um Interkulturelle Öffnung - nicht um Diversität - und Menschen mit Migrationshintergrund oder Geflüchtete galten als Hauptzielgruppen, vor allem für klassische Leseförderung. Viele Öffentlichkeitsarbeiter`innen wissen also, wen sie erreichen wollen und fragen nach den Barrieren. Zumeist wird Sprache als Barriere identifiziert und auf mehrsprachige Flyer gesetzt. Wenn ich Sie richtig verstehe, dann muss man aber weiter gehen und die Kommunikation mit den Communitys suchen. Dort nachfragen, wo sie die eigentlichen Barrieren sehen.

Prasanna Oommen: Das ist das Stichwort, wie Sie es richtig sagen, und meine absolute Überzeugung. Wenn ich im Beratungskontext unterwegs bin, dann sage ich immer: Das Wissen ist längst da. Eine der wichtigsten Aufgaben in diesem Kontext ist Recherche. Das spielt in vielen Institutionen viel zu wenig eine Rolle. Recherche bedeutet zum einen Street-Research, also wirklich rausgehen aus der Institution. Damit ist auch der Begriff der aufsuchenden Kulturarbeit gemeint. Aber es geht auch nicht unbedingt darum, diejenigen, die noch nicht da sind, davon zu überzeugen, dass ich ihnen etwas vermitteln muss. Sondern es geht eigentlich um die Haltung, dass wir in unserer Institution vielleicht auch Wissen noch nicht repräsentiert haben, was wir aber dringend brauchen, um die Stadtgesellschaft zu verstehen.

Das Thema der Öffentlichkeitsarbeit in den Bibliotheken, insbesondere Vermittlungsformate für Deutsch, möchte ich gern noch einmal aufgreifen. Diese sehe ich ein bisschen losgelöst. Wenn es sich um erfolgserprobte Formate handelt, macht es auch manchmal Sinn, an den Werbetools, also wie man diese Veranstaltungen oder Formate bewirbt, festzuhalten. Im Moment wage ich zu behaupten, dass wir aber darüber hinausgehen müssen. Zum einen stellen sich generell Fragen zur Nachhaltigkeit: Im Moment ist Drucken wahnsinnig günstig. Das heißt, wir können 10000 Flyer für einen besseren Preis als 500 Stück herstellen lassen. Da frage ich mich: Welche Verantwortung haben auch Institutionen für die Umwelt? Das andere ist: Was würde ich über digitale Formate, vielleicht auch über Face-to-face-Informationen, über Schlüsselpersonen, die in meiner Institution arbeiten, erreichen? Was ist mit Multiplikator`innen und Mitarbeiter^innen, die für ihre Communitys erkennbar sind, für jemanden mit einem vielleicht muslimischen, asiatischen oder afrikanischen Hintergrund? Wenn Menschen sehen, da ist eine Person, die kommt mir bekannt vor, mit der rede ich gerne mal kurz darüber oder die spricht vielleicht Französisch oder Arabisch? Es ist zwar einfach zu sagen, wir drucken mehrsprachige Flyer. Aber 
oft ist das nicht die Lösung für die Gewinnung eines neuen Publikums. Und das wissen die meisten Institutionen mittlerweile.

Viele sind schon an dem Punkt, zu sagen: Irgendwie haben wir trotzdem kein anderes Publikum in unserer Bibliothek. Eine ganz große Barriere bei Bibliotheken oder überhaupt bei Kultureinrichtungen, die ein Dritter Ort sein wollen also dieser Ort, der eben neben dem Arbeitsplatz und dem Privatem existiert sind Öffnungszeiten. Ich lebe ja in Köln und die Zentralbibliothek ist endlich sonntags geöffnet. Das hat lange, lange gedauert und ging vorbei an den Lebensrealitäten der Menschen. Ich hatte letztes Jahr das Glück, Marie Oestergaard, die Projektleiterin der Bibliothek Aarhus ${ }^{2}$ in Dänemark, auf meinem Podium zu haben. Danach lud sie mich ein und wir haben uns im Sommer 2019 die Bibliothek angesehen. Dort haben sie zehn Jahre mit der Zivilgesellschaft und der Stadtverwaltung in verschiedenen Arbeitsgruppen ein Konzept erarbeitet und so die Bibliothek zum Zentrum der Stadt gemacht. Natürlich gab es viele finanzielle Mittel in Aarhus als zweitgrößte Stadt Dänemarks. Aber was besonders wichtig war und ist: Sie haben viel Expertise von außen, auch von anderen Bibliotheken eingeholt. Das ist es, was mich auch in der Podiumsdiskussion in der Kunstsammlung NRW mit Marie Oestergaard zum Thema Dritter Ort überzeugt hat: das Motiv der gegenseitigen Verbindlichkeit. Also zu sagen: Wir als Institution geben bestimmten gesellschaftlichen Gruppen Raum, also einfach praktisch Räume. Dafür müssen diese nichts bezahlen. Sie nannte das Beispiel von einer Toddlers Fathers Group, also von jungen Vätern in Elternzeit, die nach einem Raum gefragt haben, wo sie sich treffen können. Die Bibliothek hat ihnen einen Raum gestellt, aber im Gegenzug verlangt, dass sich die Männer einbringen und ein Vermittlungsformat gestalten, bspw. einen Vortrag oder Wickelkurs. Es sollte also eine Exchange-Leistung erbracht werden, die der Gesellschaft vor Ort dient. Das halte ich für sehr kreativ, zu sagen, unterschiedliche Gruppen dürfen in die Institution und ein Austausch wird ermöglicht.

Julia Hauck: Öffentlichkeitsarbeit als solche erfordert ja eine gewisse Offenheit, um auf solche kreativen Ansätze zu kommen. Ich denke auch, dass es gut ist, Flyer zu übersetzen und gerade auch in Leichte Sprache, aber das allein reicht nicht, um eine neue Nutzer*innenschaft in die Bibliotheken zu locken. Es müssen alle Angebote überdacht werden und es muss versucht werden, die Institution gemeinsam mit der Stadtgesellschaft zu gestalten. Plakate in der Bibliothek allein funktionieren nicht mehr.

Prasanna Oommen: Ich würde sagen, all diese Konzepte haben ihren Sinn. Also Leichte Sprache dient dazu, zu entakademisieren. Vielerorts wird versucht,

2 Dokk1 - Aarhus: https://dokk1.dk/english. 
in der Öffentlichkeitsarbeit neue Wege zu gehen und Dinge auszuprobieren. Dieser Experimentierwille ist grundsätzlich gut. In einer Bibliothek kann auch Mehrsprachigkeit gut funktionieren, wenn die Stadtgesellschaft schon so geöffnet ist, also so verbunden ist mit der Institution, dass es tatsächlich einen Mehrwert darstellt. Aber es ändert nichts daran - wenn wir wieder auf die Zielgruppendiskussion zurückkommen -, dass viele Besucher`innen $\mathrm{zu}$ homogen hinsichtlich Alter und sozialem Hintergrund sind. So kann die Relevanz einer Institution nicht gesichert werden. Es geht hier nicht um eine rein altruistische Motivation. Sondern es geht darum, dass es öffentliche Mittel für viele Institutionen gibt, verbunden mit einem gewissen Auftrag. Dieser sollte immer wieder selbstkritisch überprüft werden. Man muss sich immer die Frage stellen: Wen hole ich ab und wen nicht? Wenn es Barrieren durch die Architektur, die Öffnungszeiten oder die Offenheit des Personals gibt, können die Kommunikationsmittel noch so leicht-/mehrsprachig sein. Es ändert zum Beispiel nichts daran, dass man die Institution als junge Mutter mit einem Kinderbetreuungsproblem nicht nutzen kann.

In meinen Workshops lasse ich die Teilnehmenden oft eine Persona entwickeln, um ihre Wunschzielgruppe zu definieren und genauer aufs Ziel zu fokussieren. Das geht dann darüber hinaus, dass beispielsweise über 19- bis 39-jährige Frauen, gebildet oder nicht, gesprochen wird. Stattdessen setzt man sich intensiv mit einer bestimmten Zielperson auseinander. Dann stellt man häufig fest, dass man vieles gar nicht weiß. Das regt meist eine weiterführende Diskussion an, die man gut nutzen kann.

Dabei darf man sich ruhig über Dinge streiten. Es herrscht viel zu viel Angst, sich mit diesen Dingen auseinanderzusetzen. Und ich glaube, ein Leitbildprozess, ein Selbstverständnis, ein Organisationsentwicklungsprozess sind die absolut unumgehbare Grundlage für eine verbesserte Öffentlichkeitsarbeit und auch für ein besseres Programm. Es geht darum, dass wir uns trauen müssen, diese Streitgespräche zu führen. Aber das setzt natürlich voraus, dass die Hierarchieebenen Vertrauen aufbauen. So können auch Menschen aus der Institution zu Wort kommen, die vielleicht über Wissen verfügen, welches in anderen Hierarchieebenen nicht vorhanden ist - sogenannte Hidden Champions. Deswegen sind Supervision oder eine Organisationsentwicklungs-Beratung oder Prozessbegleitung zwingend notwendig für diese Prozesse.

Julia Hauck: Es gibt schon einige Initiativen oder Projekte, an denen man sich so orientieren kann, zum Beispiel Die Neuen Deutschen Medienmacher*innen ${ }^{3}$, wenn es um konkrete Handlungsempfehlungen für eine diversitätssensible Öf-

3 Homepage des Vereins Neue Deutsche Medienmacher^innen: www.neuemedienmacher.de und das dazugehörige Glossar auf der Seite https://glossar.neuemedienmacher.de/. 
fentlichkeitsarbeit geht. Das heißt, die sich mit Bildsprache, Webseiten-Gestaltung oder Sprache beschäftigen. Momentan wird häufig über gendergerechte Sprache und Political Correctness gestritten. Was ist Ihr Eindruck dazu?

Prasanna Oommen: Worum es dabei immer geht, ist Selbstverständlichkeit und Entstereotypisierung. Auf der Seite der Neuen Deutschen Medienmache$r{ }^{\star}$ innen sieht man zig Cover des Spiegel-Magazins aus den letzten Jahrzehnten, die zeigen, wie anhand des Bildmaterials der Islam stigmatisiert wird. Wenn eine Kopftuch tragende Frau immer mit einer negativen Schlagzeile konnotiert wird, setzt sich das in meinem kulturellen Gedächtnis fest. Ähnlich verhält es sich mit Bildern, die eigentlich eine Selbstverständlichkeit verkörpern sollten, bspw. von körperlich beeinträchtigten Menschen, von queer-feministischen oder Transgender-Persönlichkeiten. All diese Menschen, die eventuell auffallen und nicht zur Norm gehören, müssen auf selbstverständlichen Positionen im Bildmaterial berücksichtigt werden. Wenn sie benutzt werden, also Tokenism dabei stattfindet, dann „verschlimmbessern“ wir die Situation. Dann heißt es einfach wieder: „Wir sind bunt.“ Das machen leider immer noch viele Kultureinrichtungen so und sie nutzen (auch aufgrund der finanziellen Ressourcen) solche Stock-Bilder, beispielsweise von einer schwarzen und einer weißen Hand oder Babys mit verschiedenen Hautfarben. Ich bin dezidiert dagegen.

Ich kann mich daran erinnern, dass ich vor vielen Jahren einen Tatort gesehen habe, in dem es eine Afrodeutsche Medizinerin namens - ich glaube - Petra Schmidt gab. Ich habe das nie vergessen, weil die Rolle einfach Petra Schmidt hieß und ihr Hintergrund nicht thematisiert wurde. Ungefähr 15 Jahre später habe ich die Schauspielerin bei einer Aufführung von Björn Bickers Urban Prayers gesehen und sie sofort erkannt. Nach der Veranstaltung konnte ich mit dem Ensemble reden und habe sie auf diese Rolle angesprochen. Sie war verwundert, dass ich mich noch daran erinnern konnte. Aber für mich war das das erste Mal im deutschen Fernsehen, dass jemand der anders aussah, mit einer Selbstverständlichkeit gezeigt wurde ohne „geothert“ zu werden ${ }^{4}$.

Wenn es um Bildmaterial geht, gibt es bereits einige Hinweise, wie man diversitätssensibel auswählen kann, bspw. über die Datenbank Gesellschaftsbilder ${ }^{5}$. Aber es ist immer noch so, dass, wenn man Diversität googelt, nach wie vor dieselben Stereotype auftauchen. Also ist die Frage, was man unter Diversität versteht. Da sind wir wieder bei der Haltung: Kann es für uns selbstverständlicher werden, dass eine Kuratorin im Rollstuhl sitzt? Kann es für uns selbstverständlicher werden, dass wir eine Leiterin des Bereichs Deutsch als Fremdsprache ha-

4 Die Schauspielerin heißt Sheri Hagen.

5 Fotodatenbank für Redaktionen, Medienmacher`innen und Blogger^innen und alle Interessierte, die für ihre Arbeit Bilder fernab von Klischees suchen: https://gesellschaftsbilder.de/. 
ben, die nicht weiß ist. Kann Vielfalt in den oberen Hierarchieebenen im Kultur- und Bildungsbereich selbstverständlich sein? Das sind die Themen. Und die Leute, die diese Selbstverständlichkeit repräsentieren, gibt es alle. Kann es für uns dauerhaft selbstverständlicher werden, dass wir in Talk-Runden Expert ${ }^{\star}$ innen für Landwirtschaft oder für Nachhaltigkeit haben, die nicht herkunftsdeutsch gelesen werden? ${ }^{6}$ Oder laden wir diese Leute weiterhin immer nur als Terrorismusexpert ${ }^{\star}$ innen oder als Integrationsexpert ${ }^{\star}$ innen ein? Wir sind in dem Moment einen Schritt weiter, wenn eine Bibliothek, auch auf Leitungsebene, von Menschen repräsentiert wird, die nicht einer heteronormativen weißen Mehrheitsgesellschaft entsprechen.

Julia Hauck: Es soll also im Prinzip um eine Form der Normalisierung gehen. In unserer Bibliothek haben wir vor kurzem selber Bildmaterial erstellt und echte Besucher^innen als Models engagiert. Wir haben quasi die normalen Fotos zum neuen Standort oder dem neuen Jugendbereich in Angriff genommen. Dafür wurden aber nicht wieder die Auszubildenden oder Mitarbeiter^innen genommen, sondern auch mal ein Mädchen mit chinesisch-deutschen Eltern, die dann aber eben nicht wieder Deutsch lernend gezeigt wird, sondern für den Jugendbereich werben soll. Es sollten Bilder entstehen, die Jugendliche ansprechen - egal welchen Herkunftshintergrunds oder Aussehens. Das ist natürlich nur ein erster Schritt, aber zumindest gibt es nun im internen Bild-Pool diversere Bilder. Wenn das von den Kolleg^innen dann weiter eingebracht und mitgedacht wird, ist es vielleicht ein Anfang. Aber auch das ist natürlich eine Haltungsfrage.

Prasanna Oommen: Ja, das ist eine Haltungsfrage. Ich glaube schon, dass so etwas wichtig ist, aber das Thema ist ein bisschen tricky. Das stell ich auch in meiner Arbeit immer wieder fest. Auf der einen Seite wollen wir eine Selbstverständlichkeit, Sie sagen Normalität, also the new normal oder unity in diversity, wie in Kanada propagiert. Es gibt aber auch einen Zwischenschritt dabei: Wir wollen parallel dazu auch die Anerkennung der Zusatzkompetenzen von Menschen, die keinen herkunftsdeutschen Hintergrund haben. Das heißt, wir kommen nie richtig weiter, wenn wir die Herkunft gar nicht mehr thematisieren. Sondern es geht im Grunde immer um die Frage: Wer spricht wie über was und wer fragt wie?

Deswegen bringe ich immer das Thema der pluralen Identität in diese Diskussion ein und frage: Können wir es schaffen, Menschen in unsere Institution einzubinden, deren Deutschsein wir nicht in Frage stellen und deren zusätzliche

6 Expertendatenbank und Recherchetool für Medienschaffende: www.vielfaltfinder.de/Vielfalt finder. 
Kompetenzen und Wissen wir gleichzeitig würdigen? Können wir jemanden vielleicht berufsbegleitend qualifizieren? Es fehlen oft ja einfach Mentor`innen oder die Netzwerke. Aber wir brauchen dieses Wissen. Man sollte das offen thematisieren und sagen: „Deine Mehrsprachigkeit, deine Expertise, deine Schlüsselfunktion, deine Brückenfunktion zu den Communitys ist für unsere Institution so wichtig." Wenn man das wertschätzt, hat man eine ganz andere Möglichkeit, zukünftige Role Models in der Institution aufzubauen. Ich bin im Rahmen meiner Medienvergangenheit Mentorin bei den Neuen Deutschen Medienmacher ${ }^{*}$ innen. Und ich kann meiner Mentee vermitteln, dass sie sich Netzwerke aufbauen kann, ohne zwingend aus einem privilegierten Elternhaus zu kommen. Ich kann das als Vorbild vermitteln und dadurch ermutigen.

Und ich kann auch vermitteln, dass es nicht funktioniert, nur aktivistisch zu argumentieren, sondern, dass ich auch die Perspektive derjenigen in den Machtfunktionen verstehen muss, weil es eben auch nicht um Exklusion von ihnen geht. Es ist enorm wichtig, die Positionen nicht untereinander auszuspielen. Ich finde in diesen Debatten, auch zum Beispiel über Gendergerechtigkeit oder das Gendersternchen, geht es immer darum, zu fragen: Was kann unser gemeinsames Ziel dabei sein? Das gemeinsame Ziel sollte sein, Selbstverständlichkeiten zu etablieren.

Julia Hauck: Das Gendersternchen ist oftmals ein Politikum in vielen Institutionen und bekommt m. E. auch manchmal ein bisschen zu viel Aufmerksamkeit. Eigentlich sollte es darum gehen, welche Haltung hinter der Entscheidung steht, möglichst inklusiv zu kommunizieren. Es hilft auch nicht, nur das Sternchen $\mathrm{zu}$ verwenden, sondern man sollte auch mit den Menschen reden, die es betrifft. Allein eine Vorgabe zu machen, dass so die neue Sprache ist, reicht am Ende nicht.

Prasanna Oommen: Im Moment nutzen das auch ganz viele Stadtverwaltungen nicht. Ich lebe auch in einer Stadt, in der die Stadtverwaltung sich nicht danach richtet. Das heißt, die Institutionen, die momentan $360^{\circ}$-Agent ${ }^{\star}$ innen haben, haben ein Problem in Köln, wenn sie ihre Kommunikation ändern wollen. Da kommen sie am Presseamt der Stadt nicht vorbei. Das Argument für Veränderung ist hier immer wieder die Selbstverständlichkeit. Ich musste mich da auch herantasten und habe viel von meinen jungen Kolleg*innen gelernt. Am Anfang hat es mich ehrlicherweise auch genervt, aber durch Diskussionen konnte ich dann verstehen, warum die jungen Frauen in meinem Team das Gendern wichtig fanden. Mittlerweile kann ich gar keinen Text mehr ohne Gendersternchen schreiben - einfach aus Gewohnheit. Das ist wie mit der Rechtschreibreform damals: Wir müssen uns an Dinge gewöhnen. Und das unterschätzen wir. Wir diskutieren das so lange platt, bis wir nicht mehr darüber 
reden, warum es eigentlich so schwer ist, Dinge zu ändern. Und dann geht es nur noch um Macht, aber nicht mehr um die Sache.

Julia Hauck: Da kann ich kurz einhaken. Es gibt in der Linguistik die Unterscheidung zwischen präskriptiver und deskriptiver Grammatik. Ich bin für den deskriptiven Ansatz, weil m. E. Sprache die Welt beschreibt und was nicht beschrieben wird, das gibt es nicht - vereinfacht gesagt. Durch das Gendern wird einfach deutlich gemacht, wo etwas fehlt, auch im Denken. Und ich glaube, dass eigentlich die Diskussion über diesen Fakt das Wichtigere ist, um irgendwann vielleicht in der Realität zu einer Gleichberechtigung zu kommen. Dass man das überhaupt einmal zeigt und zur Debatte stellt. Und dann über das Dahinter-Liegende sprechen kann. Die Sprache ist letztlich ein Mittel, um diese Themen immer wieder aufzubringen. Ich glaube, das muss man auch immer wieder mitdenken bei den Political Correctness-Debatten, wenn es darum geht, was man angeblich alles nicht mehr sagen darf. Es stellt sich die Frage: Wer ist man? Wer bestimmt jetzt, wie Sprache ist?

Prasanna Oommen: Ich glaube auch, dass diese Dinge leider sehr eng zusammen hängen. Nichtsdestotrotz kann man sich nicht davor verkriechen und sagen: „Ich kann als kleines Rädchen in der Institution sowieso nichts machen.“ Wenn wir zurück zur Öffentlichkeitsarbeit gehen, dann glaube ich, dass eine Sensibilisierung für Öffentlichkeitsarbeiter*innen unerlässlich in diesen Zeiten ist. Und zwar auch, um zu schlichten, um die Mitarbeiter^innen zu stärken. Sie stehen wirklich zwischen den Stühlen. Was sich immer wieder in unseren Seminaren herausstellt, ist, dass diese Gräben, auch zwischen den Agent*innen und den Öffentlichkeitsarbeiter ${ }^{\star}$ innen, sehr viel damit zu tun haben, dass die einzelnen Persönlichkeiten nicht um die Situation und die Bedarfe der jeweilig anderen wissen. Wenn man einen Zehn-Punkte-Plan hätte, dann würde es auch darum gehen, die Öffentlichkeitsarbeit gemeinsam mit der Leitungsebene zu sensibilisieren und zu stärken.

Was das für einen Mehrwert für die Gewinnung von sogenannten neuen Zielgruppen hat, kann man an den Good Practices im internationalen Vergleich sehen, z. B. in London in den Tate-Museen oder in skandinavischen Institutionen. Sie müssen noch einmal zurück zum eigentlichen Auftrag. Das eine ist die institutionelle Perspektive, die die/der Öffentlichkeitsarbeiter^in vertreten muss. Das ist auch richtig, aber es darf sich nicht verselbstständigen. Die/der Öffentlichkeitsarbeiter`in hat auch die Verpflichtung, die Geschäftsführung im Zweifelsfall zu beraten. Diese Beratungsfunktion haben nun in 39 Institutionen die $360^{\circ}$-Agent ${ }^{\star}$ innen, aber eigentlich auch jede ${ }^{\star}$, der/die eine verantwortliche Funktion in einer Institution innehat. Ich beobachte oft, dass diese Beratungsnotwendigkeit in den Hierarchien irgendwann verloren geht. Aber der Begriff 
agile Organisation bedeutet, dass ein Wissenstransfer in beide Richtungen funktioniert.

Julia Hauck: Also ich kann mir vorstellen, dass einige Kolleg^innen, gerade auch in den kleinen Bibliotheken sagen: „Wir haben aber nur 30 Mitarbeiter ${ }^{\star}$ innen. Es ist nachvollziehbar, den großen Ansatz zu diskutieren und auch auf die Organisation zu schauen, auf die Netzwerkarbeit, und dass eine Analyse wichtig ist. Aber letztlich ist das alltägliche Geschäft im Moment unser Problem und wir haben unsere Vorgaben.“

Prasanna Oommen: Das Argument höre ich häufig. Aber: Wir schauen uns immer an, wie groß oder klein ist die Institution und wo steht sie. An welchen Stellschrauben kann man drehen? Man kommt am bereichsübergreifenden Arbeiten nicht vorbei. Es muss andere Jour Fixes geben, ein anderes Programm. Da gehört die Öffentlichkeitsarbeit früh hinein. Das gilt für konfessionelle Einrichtungen, Bildungseinrichtungen genauso wie für Kultureinrichtungen. Wenn ich jemanden als Moderation oder Podiumsgäst*in für Veranstaltungen einlade, egal wie klein ich bin, muss ich einen Plan haben, was dabei zu berücksichtigen ist. Es muss Leitfäden geben, die in einer Institution gemeinsam geschrieben werden sollten. Jede Institution sollte ihre eigenen Festlegungen haben. Ich halte überhaupt nichts davon, diese Dinge auf Landes- oder kommunaler Ebene zu vermitteln. Man kann sich aber natürlich an anderen Organisationen, wie KiWit $^{7}$ oder Diversity Arts Culture ${ }^{8}$, orientieren, sich Impulse holen und dann sagen: Was passt davon für uns?

Sich auf das Alltagsgeschäft zurückzuziehen, ist der Tod einer Institution in 20 Jahren. Das kann ich als Unternehmerin auch nicht. Wir müssen uns Pausen verordnen - in der Sommerpause oder in den nicht so starken Monaten, in denen wir in Klausur gehen, um unser Programm anders aufzustellen. Das kann auch in einer Gruppe von nur fünf Kolleg^innen passieren. Dann kann das flexible Kommunikationskonzept der Öffentlichkeitsarbeit auf Grundlage eines Leitbildes oder einer Strategie entstehen. Wenn die Institution nämlich keine Strategie hat, wie soll eine Öffentlichkeitsarbeit Kommunikationsziele daraus entwickeln? Wie soll sie einen Zeitplan einhalten oder Meilensteine planen? Das geht nicht. Das heißt, diejenigen, die Programm machen, müssen gemeinsam mit der Leitungsebene planen. Und sie müssen auch gemeinsam mit der Institution erarbeiten, wo sie hinwollen, wen sie erreichen wollen, welches Programm gemacht werden soll und was als relevant erachtet wird. Dann ist die

7 Kompetenzverbund Kulturelle Integration und Wissenstransfer: www.kiwit.org.

8 Diversity Arts Culture. Berliner Projektbüro für Diversitätsentwicklung. https://diversity-artsculture.berlin/. 
Öffentlichkeitsarbeit aufgefordert, Vorschläge zu entwickeln, mit welchen Maßnahmen das erreicht werden kann. Das ist sehr operativ - gerade zum Thema Ressourcen und Alltagsgeschäft.

Julia Hauck: Was ich auch wichtig finde, sind Herausforderungen durch Hate Speech und Social Media. Es gibt auch Fälle, wo Veranstaltungen durchgeführt wurden, die einigen Menschen in der Stadtgesellschaft nicht gefallen haben. Und wenn es wirklich so ist, dass der Bereich Öffentlichkeitsarbeit eine Vorreiterrolle einnimmt, sei es mit gendersensibler Sprache oder eben in Kombination mit dem Programm, müssen diese dann auch mit der Gegenwehr umgehen. Sie sind zumeist die ersten, die über die öffentlichen Kanäle für die vertretene Haltung angegriffen werden. Es ist nicht nur wichtig, dass sensibilisiert wird, sondern dass auch die komplette Organisation dahinter stehen muss, um solche Dinge aufzufangen.

Prasanna Oommen: Da nehme ich sehr klar die Haltung ein, dass das eine Führung übernehmen muss. Da hat auch die Öffentlichkeitsarbeit wenige Möglichkeiten. Sie können das zwar steuern und sich proaktiv damit beschäftigen: Welche Vorgehensweise gibt es, wenn so etwas kommt? Wie monitoren wir unsere Social-Media-Kanäle? Aber die Leitungsebene muss eine Empfehlung für die Öffentlichkeitsarbeit geben, wie reagiert werden soll und sie muss für hundertprozentige Rückendeckung stehen. Man kann nicht genug darüber sprechen, wie sehr der psychische Druck Opfer von Hate Speech ${ }^{9}$ belastet. Da braucht es uneingeschränkte Solidarität, damit die Menschen sich nicht komplett zurückziehen. Generell gilt aber auch: Social Media kann man eben nur mit den entsprechenden personellen Ressourcen umsetzen, sonst wird das Arbeiten komplett entgrenzt.

Dann gibt es ein mehrstufiges System, über das man sich bei den Medienmacher^innen oder einschlägigen Anwältinnen informieren kann. Teilweise gibt es mittlerweile Beratungsstellen und Fördermittel dafür. Es muss ganz klar sein, welche Antworten gegeben werden, wann nicht mehr geantwortet wird, wann rechtliche Schritte eingeleitet werden und wann Polizeischutz notwendig wird. Das sind diese Stufen, die alle durchdekliniert sind. Da sollte man sich regelmäßig auf den neuesten Stand bringen. Die Neuen Deutschen Medienmache$\mathrm{r}{ }^{\star}$ innen bieten auch immer wieder digitale Veranstaltungen dazu an.

Julia Hauck: Die Bibliotheken befinden sich ja allgemein in vielen Änderungsprozessen, auch hin zum Dritten Ort. Vielerorts gibt es neue Angebote, auch was Partizipation angeht. Hier spielt m.E. das Konzept der Diversität perfekt

9 https://no-hate-speech.de/. 
hinein. Es ist also der richtige Moment für Veränderungen. Bibliotheken müssen sich dem digitalen Zeitalter stellen und auch ihre Relevanz für die und in der Stadtgesellschaft festigen. Sie sind prädestiniert dafür, unterschiedliche Menschen anzusprechen - gerade auch mit sogenannten „niedrigschwelligen“ Angeboten und kleineren Formaten. Innovation ist essenziell, da sich die Arbeit und die Art der Veranstaltungen, wie klassische Lesungen, verändern.

Prasanna Oommen: Auf jeden Fall. Wir sind oft zu behäbig, um uns auszutauschen, auch institutionsübergreifend. Wichtig ist, wie oben erwähnt, die Haltung in der Institution und dieses Recht der Gesamtgesellschaft, sich in den Institutionen bilden zu dürfen. Das ist das, was man manchmal in Erinnerung rufen muss.

\section{Weiterführende Literatur}

Allgemeine Erklärung der Menschenrechte, (1948) (UN-Vollversammlung). https://www.un. org/depts/german/menschenrechte/aemr.pdf (15.6.2021).

Bußmann, H., Gerstner-Link, C. \& Lauffer, H. (Hrsg.). (2008). Lexikon der Sprachwissenschaft: mit 14 Tabellen (4., durchges. und bibliogr. erg. Aufl.). Kröner.

Cordonier, J., della Croce, C., Menghini, M., (2019). Kulturelle Teilhabe: ein Handbuch. Nationaler Kulturdialog (Hrsg.).

El-Mafaalani, A. (2020). Mythos Bildung: die ungerechte Gesellschaft, ihr Bildungssystem und seine Zukunft (1. Aufl.). Kiepenheuer \& Witsch.

Fahrun, H., Zimmermann, N.-E. \& Skowron, E. (2015). Initiativen-Kochbuch, Engagement selbst gemacht, Einstieg ins Projektmanagement: Ein Handbuch des Theodor-Heuss-Kollegs.

brand1. Wirtschaftsmagazin. (2017). Strategie.

Gümüşay, K. (2020a). Sprache und Sein (1. Aufl.). Hanser Berlin in der Carl Hanser Verlag.

Gümüşay, K. (2020b). Die Sprachkäfige öffnen. Gedanken zur Bedeutung von „freier Rede“. APuZ, 12-13/2020, S. 4-7.

Neue Deutsche Medienmacher*innen. (2018). Voll im Bild. Workshop für diskriminierungsarme Berichterstattung. https://www.neuemedienmacher.de/wp-content/uploads/2019/05/ Voll-im-Bild-Workshopdokumentation.pdf (15.6.2021).

IQ Fachstelle Interkulturelle Kompetenzentwicklung und Antidiskriminierung. (2019). Veranstaltungen planen und durchführen. Diversity-sensibel - Nachhaltig - Inklusiv.

Goethe Universität Frankfurt am Main. (2016). Handlungsempfehlungen für eine diversitätssensible Mediensprache. https://www.uni-frankfurt.de/66760835/DiversitaetssensibleMediensprache.pdf (15.6.2021).

Hotze, S. (2020). Die Nutzung von inklusiver Sprache in der Online-Kommunikation von Bibliotheken. In Berliner Handreichungen zur Bibliotheks- und Informationswissenschaft: Bd. Heft 459. https://edoc.hu-berlin.de/bitstream/handle/18452/22591/BHR459-Hotze.pdf? sequence $=1 \&$ isAllowed=y (15.6.2021). 
Antidiskriminierungsbüro Köln. (2013). Sprache schafft Wirklichkeit. Glossar und Checkliste zum Leitfaden für einen rassismuskritischen Sprachgebrauch. https://www.oegg.de/ wp-content/uploads/2019/12/Glossar_web.pdf (15.6.2021). 